\title{
Evaluation of pattern and treatment of osteoarticular tuberculosis in central India
}

\author{
Sparsh Naik ${ }^{1}$, Rajesh Dulani ${ }^{2}$, Sohael M Khan ${ }^{3, *}$, Pradeep K Singh ${ }^{4}$, Tanmay Dua ${ }^{5}$ \\ ${ }^{1,3}$ Assistant Professor, ${ }^{2}$ Professor, ${ }^{4}$ Chief Spine Surgeon, ${ }^{5}$ Senior Resident, Dept. of Orthopaedics, ${ }^{1,3}$ JNMC, Wardha, \\ Maharashtra, ${ }^{2} \mathrm{GMC}$, Rajnangaon, Chhatisgarh, ${ }^{4}$ Dr. L H Hiranandani Hospital, Mumbai, Maharashtra, KEM Hospital, Mumbai, \\ Maharashtra, India
}

*Corresponding Author:

Email: drsohaelkhan@hotmail.com

\begin{abstract}
Introduction: Tuberculosis the global epidemic continues to be a major health problem despite availability of effective chemotherapy. The main aim of the study was to analyse the pattern, clinical findings, diagnosis, and treatment options for osteoarticular tuberculosis at Tertiary care hospital so that proper treatment guidelines can be formulated for the patient.

Materials and Methods: A prospective study was performed in the tertiary care. Patients clinically diagnosed as osteoarticular tuberculosis, all the patients who were regular on anti-tubercular treatment and follow-up and all the patients with radiological evidence matching with clinical profile were included in the study. All the patients who showed histopathological evidence in favor of osteoarticular tuberculosis were also included.

The patients with only pulmonary tuberculosis, with extra pulmonary involvement, with no osteoarticular involvement were excluded from the study.

Observation and Results: The age group of patient ranged from 1-80 years, mean age was 38.11 in males and 34.5 years in females with a total mean age of 36.96 years. Sex predominance was seen in males with $68 \%$. The distribution of the patient according to occupation farmer category showed a maximum of $56 \%$. Socio-economic class distribution according to modified kuppuswamy classification showed most patient belonged to Upper-Lower (IV) $82 \%$ and $18 \%$ belonged to lower-middle (III) category.

Conclusion: Tuberculosis of the spine is still the most preferred site of osteoarticular tuberculosis. Spine patient usually require early surgical intervention especially those with neurological deficits. Outcomes may be maximized through early detection and treatment, although adequate results can still be achieved with salvage procedures in patients presenting with late stages of disease.
\end{abstract}

Keywords: Tuberculosis, Spine, AKT, Pattern, Treatment.

\section{Introduction}

The WHO declared TB a global emergency in 1993 realizing its growing importance as public health problem. It developed the DOTS strategy (Directly Observed Treatment, short course) in 1994 as the new framework for effective TB control $^{1-4}$ with five components. The strategy has been adopted in many countries with flexibility and adaptation to the existing needs of the community. ${ }^{5,6}$

According to current estimates of WHO tuberculosis now kills 3 million people year worldwide. However it is estimated that India alone has got onefifth of the total world population of tuberculosis patients. Thus there are nearly 6 million radiologically proven cases of tuberculosis in India, and perhaps a quarter of these are sputum positive (editorial clinician 1968). Of all the patients suffering from tuberculosis nearly one to three percent has involvement of the skeletal system. $^{7}$

Magnetic resonance imaging (MRI) and computerized tomography (CT) have facilitated the preoperative diagnosis of tuberculosis of the spine, but the histopathological diagnosis is still essential. CTcontrolled biopsy and abscess drainage also aid in making the diagnosis. For accurate diagnosis to be established the tubercle bacteria must be recovered from the lesion. ${ }^{8}$
However very little research and epidemiological studies have been done on osteoarticular tuberculosis, which also has a major disease burden with significant morbidity mortality and loss of manpower. Our goal is to study the pattern, clinical findings, diagnosis, and treatment options for osteoarticular tuberculosis at Tertiary care hospital so that proper treatment guidelines can be formulated for the patient.

\section{Materials and Methods}

A prospective study was performed in the a tertiary care hospital to evaluate the demographic profile, pattern, outcome and treatment of osteoarticular tuberculosis at central India, utility of various diagnostic tools such as hematological investigations CT, X-ray, MRI, histopathology for diagnosis of osteoarticular tuberculosis. Patients clinically diagnosed as osteoarticular tuberculosis, all the patients who were regular on Anti-tubercular treatment and follow-up and all the patients with radiological evidence matching with clinical profile were included in the study. All the patients who showed histopathological evidence in favor of osteoarticular tuberculosis were also included. The patients with only pulmonary tuberculosis, with extra pulmonary involvement, with no osteoarticular involvement were excluded from the study. 


\section{Observation and Results}

The age group of patient ranged from 1-80 years, mean age was 38.11 in males and 34.5 years in females with a total mean age of 36.96 years. Sex predominance was seen in males with $68 \%$ and in females with $32 \%$. Range in males was 1-80 years and females were 12 57 years. The median age in male was 33.5 years and in female 36 years and total median age were 35 . The distribution of patients according gender category showed disease to be predominantly in male with $68 \%$ and female $32 \%$.

The distribution of the patient according to occupation farmer category showed a maximum of $56 \%$ followed by students with $16 \%$, housewife (12\%), labourer $(6 \%)$, shopkeeper $(4 \%)$, driver $(2 \%)$, butcher $(2 \%)$ and none (2\%). Socio-economic class distribution according to modified kuppuswamy classification ${ }^{9}$ showed most patient belonged to upper-lower (IV) $82 \%$ and $18 \%$ belonged to lower-middle (III) category.

SSEE score (Singh's Socio-economic emotional score ${ }^{10}$ shows majority of patients $33(66 \%)$ belonged to $7-9,15(33 \%)$ in $3-6,2(4 \%)$ in $10-12$.

Table 1: Socio-Economic Class (according to modified Kuppuswami classification)

\begin{tabular}{|l|c|c|}
\hline Class & Frequency & Percent (\%) \\
\hline Upper(i) & 0 & 0 \\
\hline Upper-middle ii) & 0 & 0 \\
\hline Lower-middle (iii) & 9 & 18 \\
\hline Upper-lower (iv) & 41 & 82 \\
\hline Lower (v) & 0 & 0 \\
\hline Total & 50 & 100 \\
\hline
\end{tabular}

A total of 50 cases were studied out of which Potts spine showed a maximum of $68 \%$ (34) followed by hip involvement $20 \%$ (10), and Potts spine at multiple level $6 \%(3 \%)$, and $2 \%$ (1) each accounted for wrist, femur and calcaneum cases.

Table 2: Pott's spine distribution chart

\begin{tabular}{|l|c|c|}
\hline Region & No. & Percent \\
\hline Cervical & 3 & $8.82 \%$ \\
\hline Cervico-dorsal & 0 & $0 \%$ \\
\hline Dorsal & 13 & $38.23 \%$ \\
\hline Dorso-lumbar & 3 & $8.82 \%$ \\
\hline
\end{tabular}

\begin{tabular}{|l|c|c|}
\hline Lumbar & 13 & $38.23 \%$ \\
\hline Lumbo-sacral & 2 & $5.88 \%$ \\
\hline Total & 34 & 100 \\
\hline
\end{tabular}

ASIA impairment scale( on follow up) most of the patient belonged to E category (no neurodeficit) $50 \%$, $16 \%$ to $\mathrm{D}$ and $2 \%$ each to A B,C. 1 case of mortality was not taken to account hence making total of 14 (28\%) patient in excluded category.

A Cross tabulation table between ASIA impairment scale (on admission) Vs ASIA impairment scale (on follow-up) for 37 patient of Potts spines shows $\mathrm{A}$ and $\mathrm{C}$ were reduced to 1 patient each, $\mathrm{B}$ remained same and no of patients in follow up were increased to 8 from 3 in D and 23 to 25 in E. There was 1 mortality as mentioned above. P-value $<0.005$ indicated that there was a statistical significance in ASIA score of patients on and follow. The graph also indicates that there was a neurogical improvement at the end of the treatment.

Types of management- Most of the patients were managed conservatively $56 \%$ and $22 \%$ required surgical intervention. 25 procedures were done out of which 32\% was antero-lateral decompression and instrumentation followed by $24 \%$ posterior decompression and instrumentation and $20 \%$ incision and drainage sos debridement, $16 \%$ girdlestone excision arthroplasty, and 4\% each wrist arthrodesis and anterior decompression and instrumentation. 3 procedures of incision and drainage followed by surgery were done on same patients of Potts spine.

$32(64 \%)$ patients took AKT-1, 4 (8\%) AKT-2, $2(4 \%)$ took AKT-4 and 1 patient (2\%) did not took AKT on admission

43 patient who took AKT-1 on admission decreased to 33 on follow-up out which 10 patient shifted to AKT-4 on follow-up. 4 patient continued to take AKT-2 from admission to follow-up. There was 1 mortality which was not included and P-value $<0.005$ indicated that there was a significant range in number of patients taking type of AKT on admission and follow-up when compared.

Table 3: Descriptive statistics for various parameters of our study

\begin{tabular}{|l|c|c|c|}
\hline Parameter & No. of patients & Minimum & Maximum \\
\hline Kuppuswami score & 50 & 7 & 14 \\
\hline SSEE score & 50 & 4 & 11 \\
\hline Duration of symptoms (months) & 50 & 0.3 & 24.0 \\
\hline ESR & 50 & 15 & 125 \\
\hline No. of days in hospitals & 50 & 6 & 71 \\
\hline VAS score (Visual Analogue Scoring) & 50 & 5 & 10 \\
\hline Total FIM Score & 50 & 18 & 124 \\
\hline
\end{tabular}



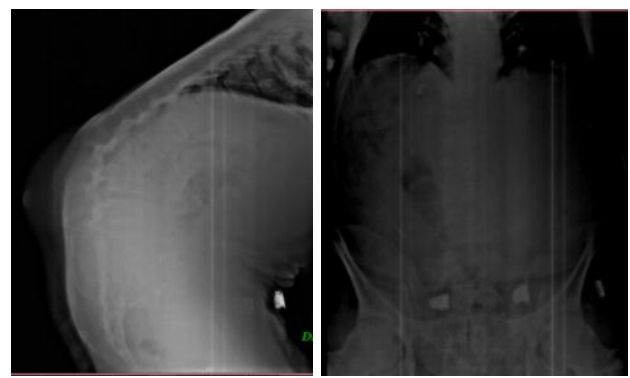

Fig. 1a: Pre-operative X-ray Ap/Lateral view
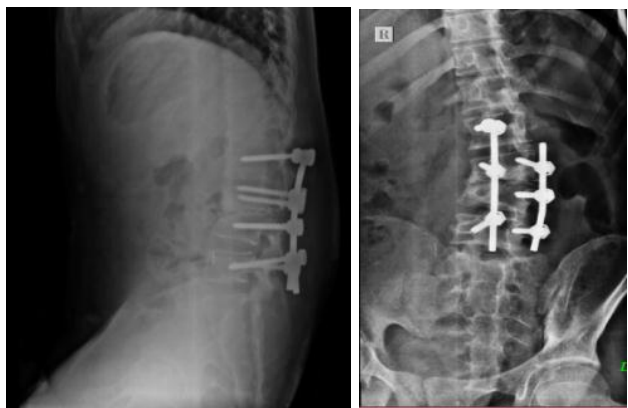

Fig. 1b: Post-operative X-ray ap/ lateral view
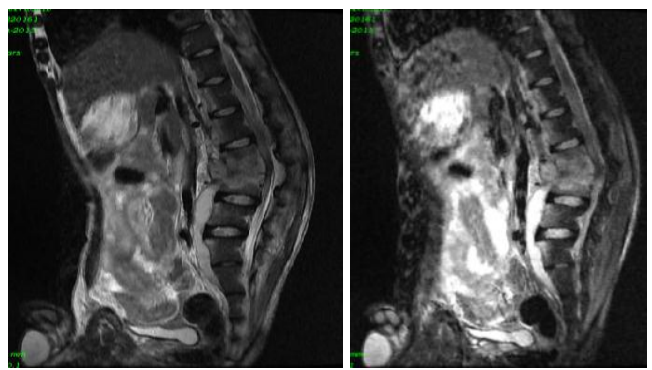

Fig. 1c: Pre-Operative MRI of LS Spine

\section{Discussion}

A total 50 cases of osteoarticular tuberculosis were studied under cross-sectional study. In our series there is a male preponderance with $68 \%$ and in female $32 \%$.

The mean age at the time of presentation in males was 38.11 years and slightly lower in female 34.5 years and total mean age in study was 36.96 yrs. The disease was reported at any age between 1- 80 yrs. The median age in male was $33.5 \mathrm{yrs}$ and $36 \mathrm{yrs}$ in females with a total median age of 35 yrs In Tuli series ${ }^{7,8}$ out of the total patients of osteoarticular tuberculosis, 52\% percent were males and $48 \%$ were females. Ranges in males were between 15-65 yrs and in female 12-70 yrs.
The median age in male was $35 \mathrm{yrs}$ and $42.5 \mathrm{yrs}$ in females with a total median age of $40 \mathrm{yrs}$.

On analyzing the age and sex distribution in patients of osteoarticular tuberculosis with other studies Sandher et $\mathrm{al}^{17}$ in his study over 79 cases found mean age 36.85 yrs (12-93 yrs) in south Asian population.

In our study modified Kuppuswamy classification ${ }^{13}$ for socio-economic class and Singh's socio-economic-emotional scale ${ }^{14}$ were taken which also takes in account the emotional support which patient gets from family member during the course of the disease. According to the modified kuppuswamy classification showed most patient belonged to upper-lower (IV) $82 \%$ and $18 \%$ belonged to lowermiddle (III) category.

Singh's socio-economic-emotional scale in which 3 being the best score and 12 worst. Most of the patient $66 \%$ in our study had a score between 7-9 with a mean value of $7.38 .^{4-11}$ Thus indicating the burden of the disease affecting the emotional health of the diseased in lower classes and lack of support from the family members. Losing wages were one of the risk factors for patients partially adhering to the DOTS treatment as seen in Gopi et $\mathrm{al}^{14}$ series. Saw Saw et $\mathrm{a}^{15}$ described that most of the patient seek free treatment for tuberculosis and refer to public sector due to costing of the drugs and its duration

Most common site of involvement was spine. ${ }^{7,15,16}$ Sandher et al ${ }^{17}$ study also showed that among osteoarticular tuberculosis, spine was the site most commonly affected (44.3\%). It can be said, if infection in spine is suspected one must rule out Pott's spine as earlier mentioned by Shaw and Thomas et al. ${ }^{20}$

In our study out of total 34 cases of spine involvement, we had cervical $(n=3)$, cervicodorsal $(n=0)$, dorsal $(n=13)$, dorsolumbar $(n=3)$, lumbar $(n=13)$, lumbosacral $(\mathrm{n}=2)$. Dorsal \&Lumbar spine involvement showed maximum with $38.23 \%$ each and followed by dorsolumbar and cervical $(8.82 \%)$ each then lumbosacral $5.88 \%$ and $0 \%$ for cervicodorsal.

Table 4:

\begin{tabular}{|l|c|c|c|}
\hline & Tuli series $^{33}$ (1974) & $\begin{array}{c}\text { Patel et al series }^{11} \\
(\mathbf{1 9 9 2 - 1 9 9 9 )}\end{array}$ & $\begin{array}{c}\text { In our series } \\
(\mathbf{2 0 1 3 - 2 0 1 5})\end{array}$ \\
\hline Cervical & $12 \%$ & $2 \%$ & $8.82 \%$ \\
\hline Cervicodorsal & $5 \%$ & - & $0 \%$ \\
\hline Dorsal & $42 \%$ & $39.16 \%$ & $38.23 \%$ \\
\hline Dorsolumbar & $12 \%$ & - & $8.82 \%$ \\
\hline Lumbar & $26 \%$ & $41.25 \%$ & $38.23 \%$ \\
\hline Lumbosacral & $3 \%$ & $16.8 \%$ & $5.88 \%$ \\
\hline
\end{tabular}


Our series (2013-2015) has shown the maximum percent of patients having dorsal and lumbar involvement. In hematological studies, the mean average ESR in our series at pre treatment was 54.37 and mean average of ESR in last follow up i.e. after 6 months was 16.37 , which was confirming the diagnosis along with other clinical findings

Patients assessed neurologically according to ASIA (American Spinal Injury Association) impairment scale from A-E where $\mathrm{A}=$ Complete paraplegia with bowel/bladder involvement and $\mathrm{E}=$ no neurodeficit. Out of 50 cases in our study, 37 patient had Potts spine (including disseminated tuberculosis cases) fourteen patients (28\%) had neurological deficit whereas twenty-three patients $(46 \%)$, Out of fourteen patients. A had three (6\%) B -1 (2\%) C-7 (14\%) and D$3(6 \%)$. The incidence of neurological impairment varies according to the series and the country concerned. In our experience, it was $28 \%$ as compared to $25 \%$ in the report of Aureganetat. ${ }^{23} \mathrm{We}$ observed reduced neurological deficit in cases of tuberculosis when compared to Eric S. Nussbaum et al's ${ }^{24}$ study.

We operated 14(28\%) of cases of Potts spine. Indication for surgery was neurological deficit in all the patients. Only 1 out 37 cases $(2.7 \%)$ was later operated after the $1^{\text {st }}$ follow-up for clinically and radiologically evident deformity of the lumbar spine leading to instability and compression over the cord.

S.M. Tuli et $\mathrm{al}^{25}$ in his study found operations for spinal tuberculosis are now indicated less for control of disease than for complications, including non responding neural deficit (nearly $40 \%$ of neural complications), prevention or correction of severe kyphotic deformity, and for tissue diagnosis (approximately 5\% of all cases). In our study also we had operated on patients with neurological deficits and spinal instability, none of the patients were operated for prevention the disease.

In our study $28(56 \%)$ patients were managed conservatively and 22 (44\%) underwent surgical procedure. Out of total surgical procedure 25,15 were operated for spine, 5 with incision and drainage sos debridement which included 1 case each of tuberculosis of femur and calcaneum and 3 of psoas abscess, 1 case was managed with wrist arthrodesis for wrist tuberculosis. 4 cases of tuberculosis of hip joint were operated with Girdlestone excision arthroplasty. 3 patients in our study underwent incision and drainage followed by surgical intervention for Potts spine accounting for 25 procedures in 22 patients.

In our study of the total spinal procedures $(30 \%)$ done $16 \%$ were antero-lateral decompression, $12 \%$ posterior decompression and 2\% anterior decompression. Either Spinal Instrumentation or fusion was done in all the procedures.

According to Sandher et $a{ }^{17}$ Surgical stabilization and/or decompression was performed in $23 \%$ of these cases because of cord compression on imaging or the presence of neurological signs which in our study was $30 \%$ slightly higher. Cui $\mathrm{X}$ et $\mathrm{al}^{26}$ in his study concluded that the posterior approach was superior to anterior instrumentation for correcting deformity and maintaining the correction.

In our study there were $10(20 \%)$ of cases of TB hip all of them were initially managed conservatively with ATT, analgesics, physiotherapy and traction out of which $4(8 \%)$ underwent girdlestone excision arthroplasty.

Sidhu AS et $\mathrm{al}^{27}{ }^{27}$ Yong qing Wang et $\mathrm{al}^{28}$, Kim et $\mathrm{al}^{29}$ in their respective studies also concluded that THR in patients with active TB of the hip is a safe procedure. If undertaken in association with extensive debridement and appropriate anti-TB treatment, it provides symptomatic relief and functional improvement. But in our study none of the patient with TB hip was managed with hip-arthroplasty.

There is controversy in the literature about the necessity of additional surgical intervention to spinal tuberculosis treatments. This difference of opinion goes back to 1960 when Hodgson ${ }^{30}$ advocated surgical treatment, and Konstam ${ }^{31}$ and colleagues advocated conservative treatment. Conservative treatment consists of only medication and sometimes-additional nonoperative measures (physical therapy, orthosis, and traction and bed rest). In our study $86 \%$ of cases were treated with CAT I, $8 \%$ with CAT II, $4 \%$ cases AKT-4 according to DOTS regimen on presentation 1 case did not take AKT due to severely deranged hepatic enzyme assay and active hepatitis and was not willing for chemotherapy subsequently later on readmission AKT was started. On follow- up after 6 months it was noted that $64 \%$ of our patients had AKT-1, $10 \%$ were taking AKT-2 whereas $24 \%$ of our patients were on AKT-4. There was 1 death noted in our series. Duration of treatment was minimum 6 months and maximum was 18 months.

Eric S. Nussbaum ${ }^{24}$ also have concluded that patients should receive at least 12 months of appropriate antituberculous therapy and individuals with neurological deficit should undergo surgical decompression Turgut $\mathrm{M}^{33}$ concluded that decompressive surgery plus anti-TB chemotherapy remains the best mode of therapy for Pott's disease

Our study matched with most of the above mentioned series and WHO guidelines that 12 months of chemotherapy along with surgery when indicated remains the cornerstone of treatment of osteoarticular tuberculosis. As Saw Saw et $\mathrm{al}^{15}$ in his study found most patients viewed the public health sector as a place to obtain free treatment and the private sector as a feepaying, convenient and better place to seek treatment. Since in our study most of the patients belonged to low socio-economic class hence most of them took AKT from DOTS center and those didn't get shifted to AKT4 after proper counseling and guidance. 
There was $1(2 \%)$ mortality noted in our series and that too after the patient was discharged from hospital as compared to $59(19 \%)$ in Nara Kingkaew, et al ${ }^{34}$ case series.

In our series the functional outcome of all patients were assessed under functional independence measure. Each patient was scored under physical and cognitive scales out of 18 items. 13 items were of physical domain and 5 of cognitive scale, which was scored from the Barthel Index. A significant statistical difference was seen in patients in pre-FIM and at follow up FIM after 6months in all physical scales. $(\mathrm{p}<0.05)$ The average follow up FIM score of 108.27 after treatment (6 months) and the pre FIM score was 101.53 showed a significant difference which indicated that the treatment was effective among our patients who was under antituberculous chemotherapy and underwent surgery. Also the effectiveness of the treatment in our study can be measured by significant statistical difference in mean preoperative VAS score which was 7.41 and at last follow up which was 2.41 with $p$ value less than 0.05 .

\section{Conclusion}

Osteoarticular Tuberculosis remains a significant fraction of the total tuberculosis cases all over the world especially in India, which has the world's $2^{\text {nd }}$ largest population and has the highest prevalence of tuberculosis. DOTS strategy is as effective as shortcourse daily regimen in early stages of disease. Clinical outcome of treatment depends on the amount of destruction at the commencement of chemotherapy, with excellent results in the predestructive and early destructive phases of disease. Spine patient usually require early surgical intervention especially those with neurological deficits. Indications for surgery can be concised to lack of response to chemotherapy, profound or progressive neurologic deficit, mechanical instability, or progression of deformity. Outcomes may be maximized through early detection and treatment, although adequate results can still be achieved with salvage procedures in patients presenting with late stages of disease.

\section{References}

1. Raviglione MC, Pio A. Evolution of WHO policies for tuberculosis control, 1948- 2001. Lancet 2002; 359: 775-80.

2. Garner P, Volmink J. Directly observed treatment for tuberculosis. Less faith, more science would be helpful. BMJ 2003;327: 823-4.

3. Behera D. TB Control: role of DOTS. Expert Rev Resp Med 2009; 3: 557-60.

4. Dye C, Garnett GP, Sleeman K, Williams BG. Prospects for worldwide tuberculosis control under the WHO DOTS strategy. Lancet 1998; 352:1886-91.

5. IJO Jan 2006 vol40: num 1:p1-15( World Health Organization. Community contribution to TB care: practice and policy. WHO Stop TB Department, Geneva, 2003.) (WHO/CDS/TB/2003.312).
6. IJO Jan 2006 vol40: num 1:p1-15( Maher D, Uplekar M, Blanc L, Ravglione M. Treatment of tuberculosis, Concordance is a key step. BMJ 2003; 327: 822-3.)

7. SM Tuli, Tuberculosis of the Skeletal System 3rd edition p 1-2. Jaypee Brothers.

8. Lakshanpal VP, Tuli SM, Singh Hardas, Sen PC. IJO 06.2008 vol 42 issue 2 . The value of histology, culture and guinea pig inoculation examination in osteoarticular tuberculosis. Acta Orthop Scand 1974;45:36-42.

9. BP Ravi Kumar, Shankar Reddy Dudala, AR Rao. Kuppuswamy's Socio-Economic Status Scale - A Revision Of Economic Parameter For 2012. International Journal of Research \& Development of Health. Jan 2013; Vol 1(1): 2-4

10. Nikose S, Singh P, Khan S, Arora M, Taywade S, et al. (2015) Prevalence of Osteoporosis in Female Population in Rural Central India [By Calcaneal Ultrasound]. J Women's Health Care 4: 262. doi:10.4172/21670420.1000262

11. Patel et al, IJO (2000) Vol.34 No.4, Tuberculosis of Lumbosacral junction.

12. Pun et al, Tuberculosis of the Lumbosacral junction, JBJS (1990), 72-B, 675- 678.

13. Sharma S, Gupta SK, Varshney A, Sharma A, Bansal A, Choudhary A. A Study of Osteoarticular Tuberculosis in a Tertiary Care Hospital of Bhopal, Madhya Pradesh. Natl J Community Med 2013; 4(1):117-20.

14. P.G Gopi, Risk factors for Non-adherence to Directly Observed Treatment (DOT) in rural tuberculosis unit, Indian Journal tuberculosis 2007.

15. Saw, Saw, Lenore Manderson, Mridula Bandyopadhyay, Than Tun Sein, Myo Myo Mon, and Win Maung. "Public and/or private health care: Tuberculosis patients' perspectives in Myanmar." Health Research Policy and Systems 7, no. 1(2009):19.

16. V. Sanchis-Olmos, M.D. Skeletal Tuberculosis. (Pp. 261. 27s. 6d.) London: Bailliere. Tindall and Cox. 1948

17. D. S. Sandher Bone And Joint Tuberculosis -Cases In Blackburn Between 1988 and 2005. J Bone Joint Surg Br; October 2007,Vol 89-B No.10:1379-1381.

18. Haider Abdul-Lateef Mousa. Bones and Joints Tuberculosis. Bahrain Medical Bulletin, Vol. 29, No.1, March 2007.

19. Ansari S, Amanullah M, Ahmad K, Rauniyar RK. Pott's spine: Diagnostic imaging modalities and technology advancements. North Am J Med Sci 2013;5:404-11.

20. Shaw NE, Thomas TG. Surgical treatment of chronic infective lesions of the spine. Br Med J 1963;1:162-4.

21. Kumar K, Saxena MB. Multifocal osteoarticular tuberculosis. Int Orthop. (SICOT) 1988; 12:135-138.

22. Huang CH. Extra-articular tuberculous osteomyelitis. Int Orthop (SICOT) 1996;20: 169-171.

23. Auregan $\mathrm{G}$ et al. Le Mal de Potta Djibouti. Analyse de 178cas. Medecine et Armees 1990; 18: 3-6.

24. Eric S. Nussbaum, Gaylan L. Rockswold, Thomas A. Bergman, Donald L. Erickson, Edward L. Seljeskog Spinal tuberculosis: a diagnostic and management challenge.Journal of Neurosurgery, August 1995 / Vol. 83 / No. 2:Pages 243-247.

25. S M Tuli. Tuberculosis of the Spine: A Historical Review. Clinical Orthopaedics \& Related Research: July 2007 - Volume 460-Issue-pp 29-38.

26. Cui X, Ma YZ, Chen X, Cai XJ, Li HW, Bai YB Outcomes of different surgical procedures in the treatment of spinal tuberculosis in adults. Med Princ Pract. 2013;22(4):346-50. 
27. A. S. Sidhu, A. P. Singh, A. P. Singh. Total hip replacement in active advanced tuberculous arthritis $\mathrm{J}$ Bone Joint Surg [Br] 2009;91-B:1301-4.

28. Yongqing Wang et al. Total hip arthroplasty for active tuberculosis of the hip. Int Orthop. 2010 Dec;34(8):11111114.

29. Kim SJ, Postigo R, Koo S, Kim JH. Total hip replacement for patients with active tuberculosis of the hip: A systematic review and pooled analysis. Bone Joint J.2013;95-B:578-82.

30. Hodgson and Stock41 1956 Anterior spinal fusion the operative approach and pathological findings in 412 patients with pott's disease of the spine Article first published online: 6 DEC 2005

DOI:10.1002/bjs.18004820819 British Journal of Surgery Volume 48, Issue 208, pages 172-178, September 1960.

31. Konstam, P.G. Spinal tuberculosis in Nigeria. Ann.R.Coll.Surg.Engl,32,99-114,1963.

32. Ravindra Kumar Garg, Dilip Singh Somvanshi. Spinal tuberculosis: A review. The Journal of Spinal Cord Medicine 2011 Vol. 34 No. 5.

33. Turgut M Spinal tuberculosis (Pott's disease): its clinical presentation, surgical management, and outcome. A survey study on 694 patients. Neurosurg Rev. 2001 Mar;24(1):8-13.

34. Nara Kingkaew, Burachat Sangtong, Waraya Amnuaiphon et al. HIV-associated extrapulmonary tuberculosis in Thailand: epidemiol ogy and risk factors for death. International Journal of Infectious Diseases (2009) 13,722-729. 